Table 1. Distribution of KL grades in the training and testing sets.

\begin{tabular}{lcccccc}
\hline KL grades & 0 & 1 & 2 & 3 & 4 & Total \\
\hline Training set & 10893 & 4582 & 6114 & 3320 & 799 & 25,978 \\
& $41.9 \%$ & $18.7 \%$ & $23.5 \%$ & $12.8 \%$ & $3.1 \%$ & \\
Testing set & 2472 & 1353 & 1696 & 775 & 194 & 6,490 \\
& $38.1 \%$ & $20.8 \%$ & $26.1 \%$ & $11.9 \%$ & $3.0 \%$ & \\
\hline
\end{tabular}

Table 2. Performance matrices of the Deep-Ten and ResNet18 models to detect osteoarthritis

\begin{tabular}{lcc}
\hline & Deep-TEN & ResNet18 \\
\hline Sensitivity & $62.29 \%$ & $59.14 \%$ \\
Specificity & $(95 \% \mathrm{Cl}, 60.42 \%-64.13 \%)$ & $(95 \% \mathrm{Cl}, 57.24 \%-61.01 \%)$ \\
& $90.07 \%$ & $94.09 \%$ \\
Positive predictive value & $(95 \% \mathrm{Cl}, 89.07 \%-91.00 \%)$ & $(95 \% \mathrm{Cl}, 93.30 \%-94.82 \%)$ \\
& $81.37 \%$ & $87.46 \%$ \\
Negative predictive value & $(95 \% \mathrm{Cl}, 79.81 \%-82.84 \%)$ & $(95 \% \mathrm{Cl}, 85.96 \%-88.82 \%)$ \\
& $(95 \% \mathrm{Cl}, 77.64 \%-79.65 \%)$ & $(95 \% \mathrm{Cl}, 75.93 \%-77.59 \%)$ \\
\hline
\end{tabular}

Conclusion: This study demonstrates that the bone texture model performs reasonably well to detect radiographic osteoarthritis with a similar performance to the bone contour model.

References:

[1] Bertalan Z, Ljuhar R, Norman B, et al. Combining fractal- and entropy-based bone texture analysis for the prediction of osteoarthritis: data from the multicenter osteoarthritis study (MOST). Osteoarthritis Cartilage 2018;26:S49.

[2] Lindner C, Wang CW, Huang CT, et al. Fully Automatic System for Accurate Localisation and Analysis of Cephalometric Landmarks in Lateral Cephalograms. Sci Rep 2016;6:33581.

[3] Zhang H, Xue J, Dana K. Deep TEN: Texture Encoding Network. The IEEE Conference on Computer Vision and Pattern Recognition (CVPR) 2017:708-17.

Disclosure of Interests: None declared

DOI: 10.1136/annrheumdis-2020-eular.2858

\section{OP0063 QUANTITATIVE COMPUTED TOMOGRAPHY PREDICTS 10-YEAR MORTALITY IN INTERSTITIAL LUNG DISEASE RELATED TO SYSTEMIC SCLEROSIS}

A. Ariani ${ }^{1}$, E. Bravi ${ }^{2}$, M. De Santis ${ }^{3}$, V. Hax $^{4}$, S. Parisi ${ }^{5}$, F. Lumetti ${ }^{6}$, F. Girelli ${ }^{7}$, M. Saracco ${ }^{8}$, F. De Gennaro ${ }^{9}$, A. Giollo ${ }^{10}$, M. Abdel Jaber ${ }^{11}$, F. Bozzao ${ }^{12}$, M. Silva ${ }^{13}$, M. C. Ditto ${ }^{5}$, C. Lomater ${ }^{8}$, F. Mozzani ${ }^{1}$, D. Santilli' ${ }^{1}$, E. Di Donato ${ }^{1}$, A. Becciolini ${ }^{1}$, F. Pucciarini ${ }^{2}$, L. Canziani ${ }^{3}$, F. C. Bodini ${ }^{14}$, E. Arrigoni ${ }^{2}$, M. Bredemeier ${ }^{15}$, R. Mendonça Da Silva Chakr ${ }^{4}$, A. Spinella ${ }^{6}$, L. Idolazzi ${ }^{10}$, R. Bortolotti ${ }^{11}$, P. Tomietto ${ }^{12}$, E. Baratella ${ }^{16}$, S. Tollot $^{16}$, D. Giuggioli ${ }^{6}$, F. Fischetti $1^{12}$, E. Fusaro ${ }^{5}$, N. Sverzellati ${ }^{13}$, C. A. Scirè ${ }^{17} .{ }^{1}$ AOU, Internal Medicine and Rheumatology Unit, Parma, Italy; ${ }^{2}$ Ospedale G. Da Saliceto, Internal Medicine and Rheumatology Unit, Piacenza, Italy: ${ }^{3}$ Humanitas Clinical and Research Center, Rheumatology and Clinical Immunology, Rozzano, Italy; ${ }^{4}$ University of Rio Grande do Sul, Hospital de Clínicas, Division of Rheumatology, Porto Alegre, Brazil; ${ }^{5} \mathrm{AOU}$ Città della Salute e della Scienza, Rheumatology Department, Turin, Italy; ${ }^{6}$ University of Modena and Reggio Emilia, AOU, Policlinico, Rheumatology Unit, Modena, Italy; ${ }^{7}$ Ospedale GB Morgagni - AUSL, Internal Medicine Unit-Rheumatology Service, Forli, Italy; ${ }^{8}$ Ospedale Mauriziano - Umberto I, Rheumatology Unit, Turin, Italy; ${ }^{9} \mathrm{AO}$ "Istituti Ospitalieri", Rheumatology Unit, Cremona, Italy; ${ }^{10}$ University of Verona, AO, Rheumatology Unit, Verona, Italy; ${ }^{11}$ Ospedale Santa Chiara, Rheumatology Unit, Trento, Italy; ${ }^{12}$ University of Trieste, Cattinara Teaching Hospital, Department of Medical Sciences, Trieste, Italy; ${ }^{13}$ University of Parma, Unit of Surgical Sciences, Section of Radiology, Parma, Italy; ${ }^{14}$ Ospedale G. Da Saliceto, Radiology Unit, Piacenza, Italy; ${ }^{15}$ Hospital Nossa Senhora da Conceição, Rheumatology Service, Porto Alegre, Brazil; ${ }^{16}$ ASUI, Department of Radiology, Trieste, Italy; ${ }^{17}$ University of Ferrara - AOU Sant'Anna, Section of Rheumatology, Ferrara, Italy

Background: Interstitial lung disease (ILD) is the main cause of death in Systemic Sclerosis (SSc). Chest CT is the gold standard in detecting ILD although it is not easy to understand its prognostic value. ILD qualitative assessment is almost worthless. Goh et al. semi quantitative score of ILD extent is related to mortality risk but it is burdened by relevant inter/intra-readers variability. An operator independent algorithm based on voxel-wise analysis proved to identify SSc patients with an increased risk of mortality according to prediction models.
Objectives: To verify if quantitative analysis of chest CT (QCT) predict 10 years-mortality in SSc patients.

Methods: SSc patients with availability of a chest CT were enrolled in 13 different centers. The CT voxel-wise analysis with a free software (www.horosproject. com) provided QCT indexes: kurtosis, skewness, mean lung attenuation and standard deviation. Patients characteristics, autoimmune profile and pulmonary function test were collected. The follow-up interval lasted from the date of ches CT to the one of the last visit or death. Each QCT index cutoff, established in a previous study (1), clustered patients in two groups. Kaplan-Meier analysis estimated and compared survival in the above mentioned groups. $p<0.05$ was considered statistically significant.

Results: Five hundred sixty three SSc patients were enrolled (35938 patientmonths); $52.4 \%$ had ILD detectable at CT scan. For each QCT index cutoff the cohort was split in two subgroups without differences in terms of sex, age, disease duration, autoimmune profile. All QCT indexes' cutoff selected subgroups with statistically different survival rate (e.g in Figure 1).

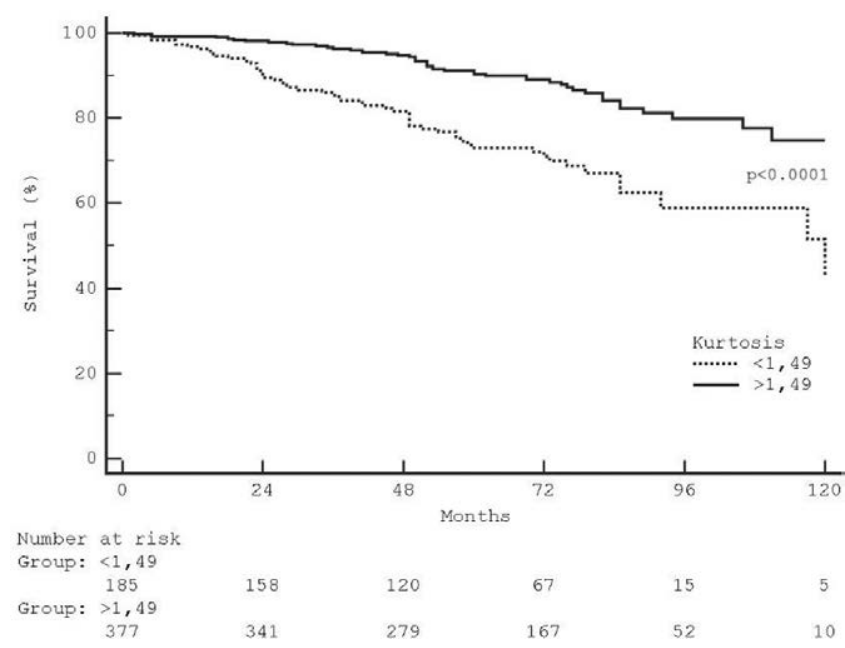

Figure 1

Conclusion: QCT can arise as the new gold standard in identifying SSc patients with poor prognosis. The real possibility to stratify SSc subjects according mortality risk will have a pivotal role in ILD treatment decisional process with the incoming anti-fibrotic drugs.

References:

[1] Ariani A et al. Rheumatology 2017

Disclosure of Interests: Alarico Ariani: None declared, Elena Bravi: None declared, Maria De Santis: None declared, Vanessa Hax: None declared, Simone Parisi: None declared, Federica Lumetti: None declared, Francesco Girelli: None declared, Marta Saracco: None declared, Fabio De Gennaro: None declared Alessandro Giollo: None declared, Masen Abdel Jaber: None declared, Francesco Bozzao: None declared, Mario Silva: None declared, Maria Chiara Ditto: None declared, Claudia Lomater: None declared, Flavio Mozzani: None declared, Daniele Santilli: None declared, eleonora Di Donato: None declared, Andrea Becciolini Speakers bureau: Sanofi-Genzyme, UCB and AbbVie, Francesco Pucciarini: None declared, Lorenzo Canziani: None declared, Flavio Cesare Bodini: None declared, eugenio arrigoni: None declared, M Bredemeier: None declared, Rafael Mendonça da Silva Chakr: None declared, Amelia Spinella: None declared, Luca Idolazzi: None declared, Roberto Bortolotti: None declared, Paola Tomietto: None declared, Elisa Baratella: None declared, Saverio Tollot: None declared, Dilia Giuggioli: None declared, Fabio Fischetti: None declared, Enrico Fusaro: None declared, Nicola Sverzellati: None declared, Carlo Alberto Scirè: None declared DOI: 10.1136/annrheumdis-2020-eular.2239

\section{OP0064 AUTOMATIC SCORING OF ARTHRITIS DISEASE ACTIVITY ON ULTRASOUND IMAGES FROM RHEUMATOID ARTHRITIS PATIENTS WITH CASCADED CONVOLUTIONAL NEURAL NETWORKS}

A. Christensen ${ }^{1}$, S. A. Just ${ }^{2}$, J. K. H. Andersen ${ }^{1}$, T. R. Savarimuthu ${ }^{1}{ }^{1}{ }^{\text {University }}$ of Southern Denmark, Maersk Mc-Kinney Moller Institute, Odense, Denmark; ${ }^{2}$ Odense University Hospital, Svendborg Hospital - Department of Medicine, Section of Rheumatology, Svendborg, Denmark

Background: Systematic Power or Color Doppler (CD) ultrasound (US) of joints can be used for early detection of Rheumatoid Arthritis (RA), predicting radiographic progression and early detection of disease flare in established 
RA [1, 2]. The international standard for performing RA US scanning and evaluation of disease activity is the OMERACT-ELUAR Synovitis Scoring (OESS) system [1, 3].

To further mitigate the operator-dependency in scoring disease activity on CD US images in future trials and clinical practice, we proposed the use of convolutional neural networks (CNN) to automatically grade CD US images according to the OESS definitions. This study is a continuation of the findings in our previous work, where we developed a CNN for four-class CD US OESS scoring with a test accuracy of $75.0 \%$ [4]

Objectives: Since our last contribution, we have further developed the architecture of this neural network and can here present a new idea applying a Cascaded Convolutional Neural Network design. We evaluate the generalizability of this method on unseen data, comparing the $\mathrm{CNN}$ with an expert rheumatologist.

Methods: The images used for developing the algorithms were graded by a single expert rheumatologist according to the OESS system. The CNNs in the cascade were trained individually, after which they were combined to form the cascade model as shown in figure 1 . The algorithms were evaluated on a separate test dataset, which came from the same distribution as the training dataset. The algorithms were compared to the gradings of an expert rheumatologist on a per-joint basis using a Kappa test, and on a per-patient basis using a Wilcoxon Signed Rank test.

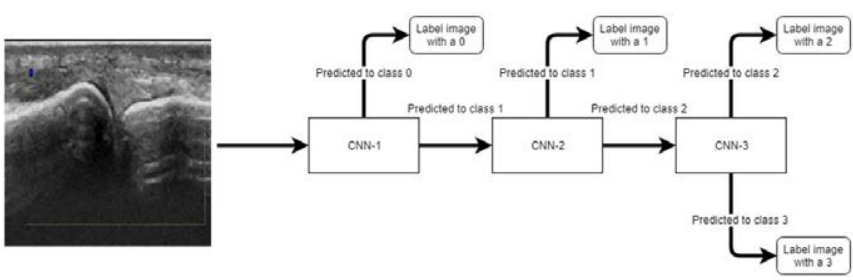

Figure 1. CNN-1 is the first CNN in the model and distinguishes between RA disease grade (DG) 0 and DG's 1, 2 and 3. CNN-2 is the second CNN and distinguishes between DG 1 and DG's 2 and 3 . CNN-3 is the final CNN which distinguishes between DG's 2 and 3.

Results: With 1678 images available for training and 322 images for testing the model, the model achieved an overall 4 -class accuracy of $83.9 \%$. On a per-patient level, there was no significant difference between the classifications of the model and of a human expert $(\mathrm{p}=0.85)$.

Conclusion: We have shown that dividing a four-degree classification task into three successive binary classification tasks has resulted in a model capable of making correct classifications in $83.9 \%$ of the cases for a test set of ultrasound images with a naturally occurring distribution of RA joint disease activity scores. Furthermore, we have shown that the cascade model can produce classification decisions comparable with a human rheumatologist when applied on a per-patient basis. This emphasizes the potential of using CNNs with this architecture as a strong assistive tool for the objective assessment of disease activity of RA patients. References:

[1] D'Agostino M-A, Terslev L, Aegerter P, et al. (2017). Scoring ultrasound synovitis in rheumatoid arthritis: a OMERACT-EULAR ultrasound taskforce-Part 1: definition and development of a standardised, consensus-based scoring system. RMD Open.

[2] Paulshus NS, Aga A-B, Olsen I, et al. (2018). Clinical and ultrasound remission after 6 months of treat-to-target therapy in early rheumatoid arthritis: associations to future good radiographic and physical outcomes. Ann Rheum Dis, 77, s. 1425-25

[3] Terslev L, Naredo E, Aegerter P, et al. (3 2017). Scoring ultrasound synovitis in rheumatoid arthritis: a OMERACT-EULAR ultrasound taskforce-Part 2: reliability and application to multiple joints of a standardised consensus-based scoring system. RMD Open.

[4] Andersen JKH, Pedersen JS, Laursen MS, et al. Neural networks for automatic scoring of arthritis disease activity on ultrasound images. RMD Open 2019; 5:e000891. doi:10.1136/ rmdopen-2018-000891

Disclosure of Interests: None declared

DOI: 10.1136/annrheumdis-2020-eular.888

\section{\begin{tabular}{|l|l}
\hline OP0065 INTER-RATER RELIABILITY AND VALIDITY OF \\
\hline
\end{tabular} MEDIAL AND LATERAL FEMORAL BONE MARROW LESIONS IN PATIENTS WITH OSTEOARTHRITIS UNDERGOING TIBIAL OSTEOTOMY USING THE KNEE INFLAMMATION MRI SCORING SYSTEM (KIMRISS)}

J. Schulz ${ }^{1,2}$, T. Birmingham ${ }^{1,2}$, W. P. Maksymowych ${ }^{3}$, R. G. Lambert ${ }^{3}$, S. Pritchett ${ }^{4}$, F. Beier ${ }^{2,5}$, J. R. Giffin ${ }^{2,6,7}$, T. Appleton ${ }^{2,5,6}$. ' Western University, Health and Rehabilitation Sciences, London, Canada; ${ }^{2}$ Western University, Bone and Joint Institute, London, Canada; ${ }^{3}$ University of Alberta, Medicine, Edmonton, Canada; ${ }^{4}$ London Health Sciences Centre, Radiology, London,
Canada; ${ }^{5}$ Western University, Physiology and Pharmacology, London, Canada; ${ }^{6}$ Western University, Medicine, London, Canada; ${ }^{7}$ London Health Sciences Centre, Surgery, London, Canada

Background: The Knee Inflammation MRI Scoring System (KIMRISS) is a semi-quantitative grading tool (range 0-500) used to measure features of inflammation in knee osteoarthritis (OA), including compartment-specific BML scores. Objectives: To investigate; 1) Inter-rater reliability of KIMRISS BML change scores including expert readers and a trainee reader, 2) Validity of KIMRISS BML scores by comparing medial and lateral femoral compartments in surgical and contralateral limbs before and 1 year after medial opening wedge high tibia osteotomy (HTO).

Methods: We included 27 patients with varus alignment and medial compartment knee OA [preoperative Kellgren-Lawrence grades $\leq 3$, age $=53.1 \pm 5.9,22$ males $(81 \%)$, body mass index $=29.9 \pm 4.3$ ] undergoing unilateral HTO. Sagittal 2D turbo spin-echo sequences were acquired at 3-Tesla in both knees pre and 1-year post HTO after surgical removal of hardware. Femoral BML scores in the medial and lateral compartments were assessed on images blinded to limb and time by masking surgical evidence. Three reviewers independently graded the same 108 images (27 patients, 2 limbs, 2 time points). We evaluated reliability pre-to-post HTO BML change scores in the medial and lateral femur by calculating intraclass correlation coefficients (ICC) and Bland-Altman plots with $80 \%$ Limits of Agreement (LoA) for each rater pair.

Results: Means and standard deviations are shown in Figure 1. Cumulative percent distribution plots of changes after surgery show both increases and decreases in the medial and lateral femur (Figure 2). When combining all raters, the ICC $(95 \%$ confidence interval $(\mathrm{Cl}))$ for the change in BML score for the surgical knee was $0.81(0.64,0.91)$ in the medial femur and $0.73(0.49,0.87)$ in the lateral femur. $80 \%$ LOA for change in BML score in the surgical knee was -3.56 to 5.44 . ICCs and LoAs for rater pairs are in Table 1. The surgical limb medial femur BML score was significantly greater than all other scores pre and post HTO (Figure 1). There were no significant changes after HTO. The mean change $(95 \% \mathrm{Cl})$ in medial femur BML score of the surgical knee was $1.08(-1.21,3.38)$. Using the mean scores of all raters, we compared BML scores in surgical and contralateral limbs before and after HTO using a 2-way repeated measures (limb by time) ANOVA, seperately for the medial and lateral compartments.

Table 1. Intraclass Correlation Coefficients (95\% Confidence Intervals) and $\mathbf{8 0} \%$ Limits of Agreement for change in BMLs scores in the surgical knee.

\begin{tabular}{|c|c|c|c|}
\hline a) & \multicolumn{2}{|c|}{ Medial compartment } & \multirow[b]{2}{*}{3} \\
\hline & 1 & 2 & \\
\hline 1 & $0.63(0.34,0.81)$ & & \\
\hline 2 & & & \\
\hline 3 & $0.69(0.43,0.85)$ & $0.42(0.06,0.69)$ & \\
\hline \multirow[t]{2}{*}{ b) } & \multicolumn{2}{|c|}{ Lateral compartment } & \\
\hline & 1 & 2 & 3 \\
\hline \multicolumn{4}{|l|}{1} \\
\hline 2 & $0.67(0.41,0.83)$ & & \\
\hline 3 & $0.79(0.60,0.90)$ & $0.47(0.11,0.72)$ & \\
\hline \multirow[t]{2}{*}{ c) } & \multicolumn{2}{|c|}{ Medial compartment } & \\
\hline & 1 & 2 & 3 \\
\hline
\end{tabular}

\begin{tabular}{|c|c|c|c|}
\hline 1 & & & \\
\hline 3 & $-6.09,8.61$ & & \\
\hline 4 & $-8.10,9.65$ & $-6.03,7.58$ & \\
\hline d) & \multicolumn{2}{|c|}{ Lateral compartment } & \\
\hline & 1 & 2 & 3 \\
\hline
\end{tabular}

1

$2-3.49,5.41$

$3-4.73,6.99$

$-3.43,4.95$

ICCs in the medial (a) and lateral (b) compartments, and 80\% LoA in the medial (c) and latera (d) compartments. Rater 1 = trainee reader, raters 2-3 = expert readers (one MSK radiologist and one rheumatologist)

Conclusion: The KIMRISS can reliably detect differences between femoral BML scores in symptomatic and contralateral limbs, supporting the inter-rater reliability, feasibility and validity of compartment-specific BML scores.

Disclosure of Interests: Jenna Schulz: None declared, Trevor Birmingham: None declared, Walter P Maksymowych Grant/research support from: Received research and/or educational grants from Abbvie, Novartis, Pfizer, 